\title{
USO DE ESPÉCIES NATIVAS E EXÓTICAS NA RESTAURAÇÃO DE MATAS CILIARES NO ESTADO DE SÃO PAULO (1957 - 2008) ${ }^{1}$
}

\author{
Geissianny Bessão de Assis², Marcio Seiji Suganuma³, Antônio Carlos Galvão de Melo e Giselda \\ Durigan $^{4}$
}

\begin{abstract}
RESUMO - A restauração ecológica no Brasil se intensificou nas últimas décadas, passando por uma série de transformações conceituais e de paradigmas. No princípio, a restauração era conduzida para restabelecer serviços ecossistêmicos e a riqueza e a origem das espécies utilizadas não era questionada. Atualmente, plantios em alta diversidade e somente com o uso de espécies nativas são recomendados. Com o objetivo de verificar se o número de espécies nativas e exóticas utilizadas na restauração de matas ciliares tem se modificado ao longo do tempo, analisamos 44 projetos implantados de 1957 a 2008, localizados no estado de São Paulo, em região anteriormente ocupada por Floresta Estacional Semidecidual (FES). Em cada local efetuamos o levantamento das árvores plantadas em área total de $1.000 \mathrm{~m}^{2}$, subdividida em parcelas aleatoriamente distribuídas. Classificamos como exóticas todas as espécies que não ocorrem naturalmente em região de FES. O número total de espécies amostradas por local variou de 12 a 58 e registramos espécies exóticas em todos os plantios estudados. Verificamos que a riqueza de espécies plantadas aumentou, passando de 25 espécies, em média, nas décadas de 1970, 1980 e 1990, para 33 espécies entre 2000 e 2008. Porém, o aumento no número de espécies nativas foi acompanhado pelo aumento no número de espécies exóticas, que vinha decrescendo até a década de 1990. Normas voltadas à restauração parecem ter sido bem sucedidas em aumentar a diversidade dos plantios, porém espécies exóticas ainda continuam sendo utilizadas nos projetos de restauração. Para promover plantios mais adequados aos objetivos da restauração ecológica, que primem por espécies nativas, ações como a maior fiscalização na produção das mudas fornecidas pelos viveiros e treinamento adequado dos profissionais ligados à restauração ecológica se tornam necessárias.
\end{abstract}

Palavras-chave: Restauração ecológica; Área de preservação permanente (APP); Legislação ambiental.

\section{NATIVE AND EXOTIC TREE SPECIES PLANTED IN RIPARIAN FOREST RESTORATION IN THE STATE OF SÃO PAULO (1957 - 2008)}

\begin{abstract}
Ecological restoration in Brazil has intensified in recent decades, and its concepts and paradigms have progressively changed. In the first decades, the richness of species and their origin were not questionable, since the restoration was intended to restore only ecosystem services. Currently, however, high diversity is required and only native species are accepted by environmental laws. In order to verify if the numbers of native and exotic species used in ecological restoration have changed over time, we assessed 44 riparian forests undergoing restoration in the state of São Paulo. All study areas were restored from 1957 to 2008, in sites where the former vegetation was seasonal forest (FES). At each site we sampled all trees planted in a total area of $1000 \mathrm{~m}^{2}$, divided in plots randomly distributed. We classified as exotics all species whose native range is not the Seasonal Atlantic Forest. The total number of species sampled per site ranged from 12 to 58 and exotic species were recorded in all sites. The number of species recorded increased from an average of 25 species in the 1970s, 1980s and 1990s, to 33 species from 2000 to 2008. However, the number of alien species has also increased in the same period. Rules aiming at high diversity in ecological restoration may have been successful, but the use of exotic species in restoration projects have increased in recent years. Improving inspection in forest nurseries and adequate training of professionals involved in ecological restoration are recommended actions in order to avoid the use of exotic species in restoration projects.
\end{abstract}

Keywords: Ecological restoration; Permanently protected areas; Environmental legislation.

\footnotetext{
${ }^{1}$ Recebido em 21.04.2012 aceito para publicação em 06.08.2013.

${ }^{2}$ Instituto de Pesquisa Jardim Botânico do Rio de Janeiro, IP/JBRJ, Brasil. E-mail: <geissianny@gmail.com>.

${ }^{3}$ Universidade de São Paulo, Centro de Recursos Hídricos e Ecologia Aplicada, São Carlos, SP. Email: <margiosuganuma@gmail.com>.

${ }^{4}$ Floresta Estadual de Assis, Instituto Florestal, Assis, SP. E-mail: <acgmelo@gmail.com>e <giselda@femanet.com.br>.
} 


\section{INTRODUÇÃO}

A restauração ecológica objetiva auxiliar o restabelecimento da integridade de um ecossistema que foi degradado ou destruído, buscando a reconstituição do seu funcionamento, da estrutura da comunidade e da composição de espécies nativas localmente (PALMER et al., 1997; SER, 2004).

No Brasil, a consolidação da ecologia da restauração como ciência ocorreu, sobretudo, a partir das duas últimas décadas (RODRIGUES et al. 2009; OLIVEIRA; ENGEL, 2011). Nesse período muito se avançou no conhecimento das teorias ecológicas e na sua aplicação na construção de métodos aplicados à restauração, com alguns exemplos de projetos que obtiveram sucesso (NOGUEIRA, 1977; DURIGAN; DIAS, 1990) e também de outros que foram comparativamente mal sucedidos (BARBOSA et al. 2003; SOUZA; BATISTA, 2004) em atingir os objetivos da restauração.

Inicialmente as atividades de restauração eram realizadas buscando-se apenas a reconstrução de uma fisionomia florestal, sendo pouco considerados aspectos ecológicos, sucessionais ou a escolha das espécies que deveriam ser utilizadas para recuperar os ecossistemas e seu funcionamento. Assim, as primeiras tentativas de restauração resultaram em plantios de espécies arbóreas incluindo nativas e exóticas (BELLOTTO et al., 2009). A utilização de espécies exóticas na prática da restauração de ecossistemas ocorre deste o início dessas atividades no Brasil, uma vez que não existia nenhuma restrição técnica ou jurídica quanto à origem das espécies a serem plantadas. $\mathrm{O}$ uso de exóticas pode ser facilmente constatado em listas de espécies apresentadas em dissertações e teses, anais de eventos e em artigos científicos publicados no país sobre restauração florestal (PULITANO et al., 2004; MELO; DURIGAN, 2007; RODRIGUES et al., 2010).

Algumas das espécies exóticas utilizadas em plantios de restauração se tornaram espécies-problema ou mesmo invasoras, como é o caso das jaqueiras na Floresta da Tijuca (ABREU; RODRIGUES, 2010). Diante deste problema, a utilização de espécies que ocorriam naturalmente em território brasileiro passou a ser privilegiada em detrimento de espécies oriundas de outros países. A escolha das espécies "nativas brasileiras”, no entanto, não considerava a formação vegetacional e nem mesmo o bioma ou domínio fitogeográfico em que a espécie ocorria (BRANCALION et al. 2009). Do ponto de vista ecológico, não apenas espécies de outros países são consideradas exóticas, mas também qualquer espécie que esteja fora de sua região de ocorrência natural, ainda mais em se tratando de um país com dimensões continentais como o Brasil.

No estado de São Paulo, a restauração ecológica é regulamentada por normas e diretrizes legais detalhadas sobre as espécies a utilizar desde o ano de 2001 (Resolução SMA 21/2001), impondo o uso exclusivo de espécies nativas, entre outros dispositivos. A elaboração desta norma baseou-se em extenso levantamento que constatou o declínio de muitos reflorestamentos, e o insucesso foi atribuído à baixa diversidade de espécies plantadas (BARBOSA et al. 2003). Visando seu aprimoramento, a norma foi reeditada duas vezes nos anos seguintes (Resolução SMA 47/2003 e Resolução SMA 08/2008), com aumento do número mínimo de espécies exigido para a restauração e indicação de proporções entre grupos funcionais. Atualmente, plantios exclusivamente de espécies nativas de cada formação florestal, com alta diversidade, podem ser considerados dentro da lei (SÃO PAULO, 2008).

Esta legislação e sua eficácia ainda geram debates entre os legisladores, proprietários rurais e cientistas (ARONSON, 2010; BRANCALION etal. 2010; DURIGAN et al. 2010; CALMON et al. 2011; ARONSON et al. 2011). Entre outros aspectos ainda discutidos, as dificuldades na obtenção das mudas adequadas para algumas regiões e na identificação correta das espécies que serão plantadas e de sua origem fitogeográfica são apontadas entre os principais problemas de execução e fiscalização dos plantios. De fato, em muitas áreas restauradas ainda têm sido observadas espécies exóticas (DURIGAN et al., 2010) ou mesmo espécies pouco adaptadas aos ambientes em que estão sendo introduzidas, verificando-se alta mortalidade das árvores plantadas.

A introdução de espécies em ambientes onde não ocorrem naturalmente representa uma ameaça potencial à integridade dos ecossistemas, devido ao risco de que essas espécies se tornem invasoras (MACK et al., 2000; REJMÁNEK et al., 2005). Em ecossistemas naturais, espécies exóticas invasoras comumente podem competir com espécies nativas e até substituí-las (DAVIS, 2009). Assim, pelo princípio da precaução, espécies não-nativas não deveriam ser utilizadas em plantios visando à restauração de ecossistemas. 
Espécies exóticas são utilizadas com maior freqüência na recuperação ou reabilitação de áreas degradadas, uma vez que podem ser a opção mais viável para plantios em locais com alterações ambientais severas ao ponto de serem limitantes para o estabelecimento e crescimento de vegetação nativa, como é o caso de áreas mineradas (PARROTA; KNOWLES, 1999; PRACH et al., 2007). Para a conservação da biodiversidade, no entanto, o uso de espécies nativas é geralmente preferível, visando à conservação das espécies (BROCKERHOFF et al. 2008). Ainda que exista muita polêmica em torno do uso de espécies não-nativas para fins de restauração (D’ANTONIO; MEYERSON, 2002), são raros ou praticamente ausentes os registros na literatura sobre a frequência com que essas espécies são utilizadas.

Neste estudo, analisamos a riqueza e a origem das espécies que têm sido utilizadas na restauração de matas ciliares ao longo dos últimos 50 anos, para verificar: i) a frequência e a proporção com que espécies arbóreas exóticas são utilizadas nos plantios; ii) se a riqueza dos plantios tem aumentado e se o uso de espécies exóticas tem diminuído em anos mais recentes, em resposta aos avanços do conhecimento e como resultado das restrições impostas pela legislação ambiental do Estado de São Paulo.

\section{MATERIAL E MÉTODOS}

\section{1. Áreas de estudo}

Selecionamos 44 áreas de mata ciliar, com idade variando de dois a 53 anos, restauradas por meio de plantio heterogêneo de mudas de espécies arbóreas. Todas as áreas situam-se em região de domínio da Floresta Estacional Semidecidual (VELOSO et al., 1991), nas regiões leste, centro, noroeste e sudoeste do estado de São Paulo, mais especificamente nas bacias dos rios Paranapanema, Tietê, Paraná e Paraíba do Sul (Tabela 1). A maioria dos plantios encontra-se sob clima do tipo Cwa (mais quente e com estação seca definida), ou Cfa (mais fresco, sem estação seca), segundo a classificação de Köppen. A temperatura média anual na região de estudo varia de $21^{\circ} \mathrm{C}$ a $24^{\circ} \mathrm{C}$, com temperatura média mensal mínima de 10oC em alguns locais e média das máximas de até 30oC. A precipitação anual média gira em torno de 1.400 mm, podendo ser inferior a $40 \mathrm{~mm}$ nos meses mais secos, que ocorrem durante o inverno (abril a agosto).

\subsection{Amostragem da vegetação}

Os levantamentos foram realizados nos anos de 2010 e 2011. A amostragem da comunidade vegetal em cada um dos locais estudados incluiu somente as espécies arbóreas plantadas e foi efetuada em área total de $1.000 \mathrm{~m}^{2}$, subdividida emparcelas aleatoriamente distribuídas (cinco parcelas de $200 \mathrm{~m}^{2}$ no Vale do Paraíba e dez de $100 \mathrm{~m}^{2}$ no restante do estado). Espécies oriundas da regeneração natural não foram incluídas nas análises deste estudo. A distinção entre os indivíduos que foram plantados e os que eram provenientes de regeneração natural foi feita pelo reconhecimento das linhas de plantio, evidente mesmo em plantios mais antigos. Em casos excepcionais de plantio sem alinhamento (dois locais), contamos com o auxílio dos responsáveis pela execução dos projetos para identificar as árvores plantadas. As espécies observadas nas áreas de estudo foram identificadas em campo ou coletadas para identificação posterior com auxílio de literatura especializada ou mediante consulta a especialistas.

\subsection{Classificação das espécies pela origem geográfica}

Com o objetivo de verificar quais espécies foram mais utilizadas nos projetos de restauração, classificamos todas as espécies amostradas nos 44 plantios segundo a sua origem geográfica, em três categorias: a) nativas da Floresta Estacional Semidecidual (FES), região em que se encontram os plantios estudados; b) nativas de outras formações vegetacionais brasileiras, diferentes de FES; c) espécies oriundas de outros países. Essa classificação foi realizada com base na literatura, tendo sido consultados os volumes já publicados da Flora Fanerogâmica do Estado de São Paulo (WANDERLEY et al., 2001, 2002, 2003, 2005, 2007, 2009), outras publicações que trazem a região de origem das espécies (CORRÊA, 1984; LORENZI, 1992; RODRIGUES; NAVE, 2000; CARVALHO, 2003; DURIGAN, et al., 2004; RAMOS et al., 2008), a lista de espécies que acompanha a Resolução SMA 08/2008, bancos de dados on line, como o Species Link (disponível em <http:// www.splink.org.br $>$ ) e a Lista de Espécies da Flora do Brasil (<http://floradobrasil.jbrj.gov.br $>$ ).

\subsection{Análise dos Dados}

Para as análises dos dados, reunimos em um único conjunto as espécies de outras regiões vegetacionais do Brasil e as espécies oriundas de outros países, considerando todas como exóticas, por não ocorrerem 
Tabela 1 - Localização geográfica dos 44 plantios de restauração de mata ciliar estudados por município e idade/anos após o plantio.

Table 1 - Geographical location of 44 riparian forests undergoing restoration, municipality and age/years after planting.

\begin{tabular}{|c|c|c|c|}
\hline Plantio & Idade (anos) & Município & Localização (lat./long.) \\
\hline $\mathrm{P} 1$ & 2 & São José dos Campos & $23^{\circ} 06^{\prime} 49^{\prime \prime S} / 45^{\circ} 45^{\prime} 44^{\prime \prime W}$ \\
\hline $\mathrm{P} 2$ & 3 & São José dos Campos & $23^{\circ} 08^{\prime} 47^{\prime \prime S} / 45^{\circ} 51^{\prime} 59^{\prime \prime W}$ \\
\hline P 3 & 3 & 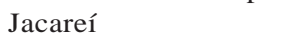 & $23^{\circ} 15^{\prime} 49^{\prime \prime S} / 45^{\circ} 59^{\prime} 08^{\prime \prime W}$ \\
\hline $\mathrm{P} 4$ & 4 & Pindamonhangaba & $22^{\circ} 52^{\prime} 19^{\prime \prime S} / 45^{\circ} 22^{\prime} 03^{\prime \prime W}$ \\
\hline $\mathrm{P} 5$ & 4 & Tremembé & $22^{\circ} 57^{\prime} 55^{\prime \prime S} / 45^{\circ} 35^{\prime} 11^{\prime \prime W}$ \\
\hline P 6 & 4 & Caçapava & $23^{\circ} 06^{\prime} 45^{\prime \prime S} / 45^{\circ} 46^{\prime} 27^{\prime \prime W}$ \\
\hline P 7 & 5 & Taubaté & $23^{\circ} 01^{\prime} 29^{\prime \prime S} / 45^{\circ} 39^{\prime} 28^{\prime \prime W}$ \\
\hline P 8 & 6 & Tremembé & 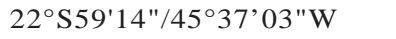 \\
\hline P9 & 6 & Tremembé & $22^{\circ} 58^{\prime} 45^{\prime \prime S} / 45^{\circ} 35^{\prime} 48^{\prime \prime W}$ \\
\hline $\mathrm{P} 10$ & 6 & Tremembé & $22^{\circ} 58^{\prime} 04^{\prime \prime S} / 45^{\circ} 33^{\prime} 54^{\prime \prime W}$ \\
\hline P 11 & 7 & Tarumã & $22^{\circ} 44^{\prime} 56,8^{\prime \prime S} / 50^{\circ} 31 ' 1,1^{\prime \prime W}$ \\
\hline $\mathrm{P} 12$ & 8 & Tremembé & $22^{\circ} 59^{\prime} 30^{\prime \prime S} / 45^{\circ} 36^{\prime} 52^{\prime \prime W}$ \\
\hline P13 & 8 & Tremembé & $22^{\circ} 58^{\prime} 32^{\prime \prime S} / 45^{\circ} 35^{\prime} 47^{\prime \prime W}$ \\
\hline $\mathrm{P} 14$ & 8 & Florínea & $22^{\circ} 48^{\prime} 40,7^{\prime \prime S} / 50^{\circ} 40^{\prime} 10,8^{\prime \prime W}$ \\
\hline P15 & 8 & Caçapava & $23^{\circ} 05^{\prime} 57^{\prime \prime S} / 45^{\circ} 43^{\prime} 31^{\prime \prime W}$ \\
\hline $\mathrm{P} 16$ & 8 & Caçapava & $23^{\circ} 05^{\prime} 45^{\prime \prime S} / 45^{\circ} 43^{\prime} 19^{\prime \prime W}$ \\
\hline $\mathrm{P} 17$ & 8 & Tremembé & $22^{\circ} 59^{\prime} 09^{\prime \prime S} / 45^{\circ} 36^{\prime} 13^{\prime \prime W}$ \\
\hline $\mathrm{P} 18$ & 9 & Tarumã & $22^{\circ} 43^{\prime} 57,4^{\prime \prime S} / 50^{\circ} 33^{\prime} 4,1^{\prime \prime W}$ \\
\hline P19 & 9 & Pindamonhangaba & $22^{\circ} 52^{\prime} 20^{\prime \prime S} / 45^{\circ} 21^{\prime} 33^{\prime \prime W}$ \\
\hline P20 & 9 & Tremembé & $22^{\circ} 58^{\prime} 02^{\prime \prime S} / 45^{\circ} 35^{\prime} 07^{\prime \prime W}$ \\
\hline P21 & 9 & Taubaté & $23^{\circ} 01^{\prime} 54^{\prime \prime S} / 45^{\circ} 39^{\prime} 11^{\prime \prime W}$ \\
\hline P22 & 10 & Tremembé & $22^{\circ} 58^{\prime} 18^{\prime \prime S} / 45^{\circ} 34^{\prime} 21^{\prime \prime W}$ \\
\hline P23 & 10 & Cândido Mota & $22^{\circ} 43^{\prime} 1,9^{\prime \prime S} / 50^{\circ} 19^{\prime} 8,7 " \mathrm{~W}$ \\
\hline P24 & 11 & Caçapava & $23^{\circ} 04^{\prime} 22^{\prime \prime S} / 45^{\circ} 41^{\prime} 21^{\prime \prime W}$ \\
\hline P25 & 11 & Jacareí & $23^{\circ} 16^{\prime} 06^{\prime \prime S} / 45^{\circ} \mathrm{W}$ 59’15"W \\
\hline P26 & 12 & Tremembé & $22^{\circ} 58^{\prime} 44^{\prime \prime S} / 45^{\circ} 35^{\prime} 52^{\prime \prime W}$ \\
\hline P 27 & 12 & Cândido Mota & $22^{\circ} 42^{\prime} 18,9^{\prime \prime S} / 50^{\circ} 21^{\prime} 59,8 " \mathrm{~W}$ \\
\hline P28 & 13 & Castilho & $20^{\circ} 45^{\prime} 36,9^{\prime \prime S} / 51^{\circ} 35^{\prime} 21,5^{\prime \prime W}$ \\
\hline P29 & 13 & Caçapava & $23^{\circ} 06^{\prime} 16^{\prime \prime S} / 45^{\circ} 46^{\prime} 32^{\prime \prime W}$ \\
\hline P30 & 14 & Tarumã & $22^{\circ} 45^{\prime} 58,9 " \mathrm{~S} / 50^{\circ} 32^{\prime} 2,1^{\prime \prime W}$ \\
\hline P31 & 14 & Jacareí & $23^{\circ} 15^{\prime} 28^{\prime \prime S} / 45^{\circ} 59^{\prime} 08^{\prime \prime W}$ \\
\hline P32 & 15 & Tremembé & $22^{\circ} 58^{\prime} 34^{\prime \prime S} / 45^{\circ} 35^{\prime} 53^{\prime \prime W}$ \\
\hline P33 & 15 & Taubaté & $22^{\circ} 59^{\prime} 50^{\prime \prime S} / 45^{\circ} 37^{\prime} 35^{\prime \prime W}$ \\
\hline P34 & 16 & Sandovalina & $22^{\circ} 32^{\prime} 7,3^{\prime \prime S} / 51^{\circ} 59^{\prime} 41,4^{\prime \prime} \mathrm{W}$ \\
\hline P35 & 17 & Sandovalina & $22^{\circ} 33^{\prime} 3,7^{\prime \prime S} / 52^{\circ} 00 ' 3,9 " W$ \\
\hline P36 & 17 & Florínea & $22^{\circ} 50^{\prime} 31,4^{\prime \prime S} / 50^{\circ} 36^{\prime} 20,1^{\prime \prime W}$ \\
\hline P37 & 18 & Tarumã & $22^{\circ} 45^{\prime} 15,1^{\prime \prime S} / 50^{\circ} 31^{\prime} 36,3^{\prime \prime W}$ \\
\hline P38 & 21 & Tarumã & $22^{\circ} 50^{\prime} 11,9 " \mathrm{~S} / 50^{\circ} 37^{\prime} 34,1^{\prime \prime} \mathrm{W}$ \\
\hline P39 & 22 & Iracemápolis & $22^{\circ} 34^{\prime} 38,5^{\prime \prime S} / 47^{\circ} 30^{\prime} 31,8^{\prime \prime} \mathrm{W}$ \\
\hline P 40 & 23 & Rosana & $22^{\circ} 35^{\prime} 14,3^{\prime \prime S} / 52^{\circ} 50 ' 52,4^{\prime \prime W}$ \\
\hline P41 & 27 & Promissão & $21^{\circ} 19^{\prime} 42,3^{\prime \prime S} / 49^{\circ} 47^{\prime} 1,7^{\prime \prime W}$ \\
\hline P42 & 28 & Cândido Mota & $22^{\circ} 46^{\prime} 56,7^{\prime \prime S} / 50^{\circ} 27^{\prime} 44,4^{\prime \prime W}$ \\
\hline P43 & 38 & Cândido Mota & $22^{\circ} 47^{\prime} 23,9 " \mathrm{~S} / 50^{\circ} 28^{\prime} 4,1^{\prime \prime W}$ \\
\hline P 44 & 53 & Cosmópolis & $22^{\circ} 40^{\prime} 19,9 " \mathrm{~S} / 47^{\circ} 12^{\prime} 27,6^{\prime \prime} \mathrm{W}$ \\
\hline
\end{tabular}

naturalmente em regiões de FES. Assim, calculamos o número e a proporção de espécies plantadas nativas e exóticas da FES e a média do número dessas espécies nas áreas de estudo. Estes dados são descritivos dos tipos de espécies que foram introduzidas nas áreas em restauração e que sobreviveram até o momento da amostragem, já que, para a maioria das áreas, não foi possível resgatar a informação precisa de todas as espécies que foram plantadas, muitas das quais não se estabeleceram nas florestas restauradas.

Revista Árvore, Viçosa-MG, v.37, n.4, p.599-609, 2013 
Para avaliar se a freqüência de uso de espécies não-nativas na restauração se modificou nos últimos 50 anos, construímos gráficos de dispersão baseados no ano de implantação dos projetos, uma vez que os plantios selecionados para estudo datam de 1957 até o ano de 2008. Calculamos o número médio de espécies nativas, exóticas e o número total de espécies plantadas nas áreas de amostragem, agrupadas por década de plantio. Os gráficos e as análises foram construídos utilizando os software OriginPro 8 (OriginLab Corporation, MA).

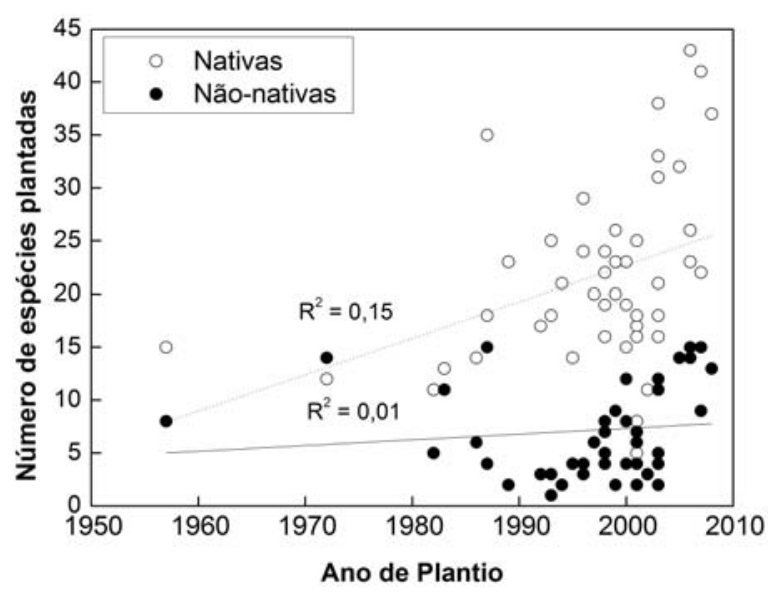

Figura 1 - Número de espécies arbóreas nativas e exóticas utilizadas nos plantios de restauração de matas ciliares no estado de SP de 1957 a $2008 . \mathrm{R}^{2}$ : coeficiente de determinação da reta.

Figure 1 - Number of native and exotic tree species planted for restoration of riparian forests in the state of São Paulo from 1957 to 2008. $R^{2}$ : determination coefficient.

\section{RESULTADOS}

Nas 44 áreas de estudo amostramos 5.394 árvores plantadas, pertencentes a 294 espécies, das quais 201 eram nativas e 93 exóticas da Floresta Estacional Semidecidual. Analisando a origem geográfica das espécies plantadas nas áreas em restauração como um todo, as espécies nativas da FES representaram $68 \%$ do total amostrado. Entre as exóticas, 51 espécies (17\% do total de espécies plantadas) eram de outras formações vegetacionais do Brasil e 42 espécies (15\% do total) eram oriundas de outros países. Dessa forma, 32\% das espécies utilizadas nos projetos de restauração não eram nativas da região em que foram plantadas.

A freqüência do uso de espécies não-nativas na restauração de matas ciliares foi elevada, sendo constatada em todos os plantios estudados (Figura 1). Porém, tanto o número total de espécies plantadas amostradas quanto o número de espécies nativas ou exóticas, foram variáveis entre as áreas (Tabela 2). Apesar do elevado número de espécies amostradas nas áreas em restauração, se consideradas espécies nativas ou não (294 espécies), a riqueza de espécies por local foi baixa, sendo registrado em média, 28 espécies plantadas em cada área, das quais sete (25\% das espécies plantadas, em média) eram exóticas da FES. A proporção de espécies exóticas em cada plantio variou de 4\% no local P35 (Sandovalina, plantio com 17 anos) a 54\% no local P3 (Cândido Mota, 38 anos). A proporção de árvores de espécies exóticas foi, em geral, inferior à proporção de espécies, variando de 1\% dos indivíduos plantados, também no local P35, até 79\% dos indivíduos plantados na área P16 (Caçapava), com oito anos.

Tabela 2 - Valores absolutos e percentuais do número médio, máximo e mínimo de espécies e de indivíduos nativos, exóticos e total, plantados em 44 áreas de restauração de mata ciliar no estado de SP, amostrados em $1000 \mathrm{~m}^{2}$ por local. A coluna “Total” corresponde aos registros dos $44.000 \mathrm{~m}^{2}$ amostrados nas 44 áreas.

Table 2 - Absolute values and percentages of average, maximum and minimum number of species and of native, exotic and total individuals planted in 44 areas of riparian forest restoration in the state of São Paulo, sampled in $1000 \mathrm{~m}^{2}$ per site. The "Total" column corresponds to the records of 44,000 $\mathrm{m}^{2}$ sampled in 44 areas.

\begin{tabular}{|c|c|c|c|c|c|c|c|c|}
\hline \multirow[b]{2}{*}{ Espécies Nativas } & \multicolumn{2}{|c|}{ Média } & \multicolumn{2}{|c|}{ Mínimo } & \multicolumn{2}{|c|}{ Máximo } & \multirow{2}{*}{\multicolumn{2}{|c|}{$\frac{\text { Total }}{(68 \%)}$}} \\
\hline & 21 & $(76 \%)$ & 5 & $(46 \%)$ & 44 & $(96 \%)$ & & \\
\hline Espécies Exóticas & 7 & $(24 \%)$ & 1 & $(4 \%)$ & 16 & $(54 \%)$ & 93 & $(32 \%)$ \\
\hline Total (espécies) & 28 & & 6 & & 58 & & 294 & \\
\hline Indivíduos Nativos & 91 & $(72 \%)$ & 13 & $(21 \%)$ & 203 & $(99 \%)$ & 4025 & $(75 \%)$ \\
\hline Indivíduos Exóticos & 31 & $(28 \%)$ & 1 & $(1 \%)$ & 90 & $(79 \%)$ & 1368 & $(25 \%)$ \\
\hline Total (indivíduos) & 122 & & 44 & & 246 & & 5397 & \\
\hline
\end{tabular}


Nenhuma espécie foi amostrada em todos os locais. No entanto, algumas espécies foram utilizadas com mais freqüência nos projetos de restauração, entre as quais se incluem as espécies nativas Citharexylum myrianthum (31 plantios), Cecropia pachystachya (30 plantios), Peltophorum dubium (29 plantios), Inga uruguensis (26 plantios), Schinus terebinthifolius (26 plantios), Ceiba speciosa (25 plantios) e Anadenanthera macrocarpa (23 plantios). Considerando-se o número de indivíduos plantados por espécie em todos os locais, as não-nativas Syzygium cumini (espécie do sul asiático) e Inga laurina (nativa da Floresta Ombrófila Densa), destacaram-se entre as dez espécies mais plantadas. Outras espécies oriundas de outros países também foram comumente encontradas nos plantios, como Psidium guajava (presente em 13 plantios), Jacaranda mimosifolia (13 plantios), Cordia myxa (12 plantios) e Leucaena leucocephala (nove plantios). Entre as espécies de outras formações vegetacionais brasileiras destaca-se o uso de Schizolobium parahyba (14 plantios) e Guazuma crinita (nove plantios), ambas nativas da Floresta Ombrófila Densa.

Por meio da análise da relação entre o uso de espécies nativas e exóticas com a idade dos plantios, observamos que, embora o tempo de implantação das

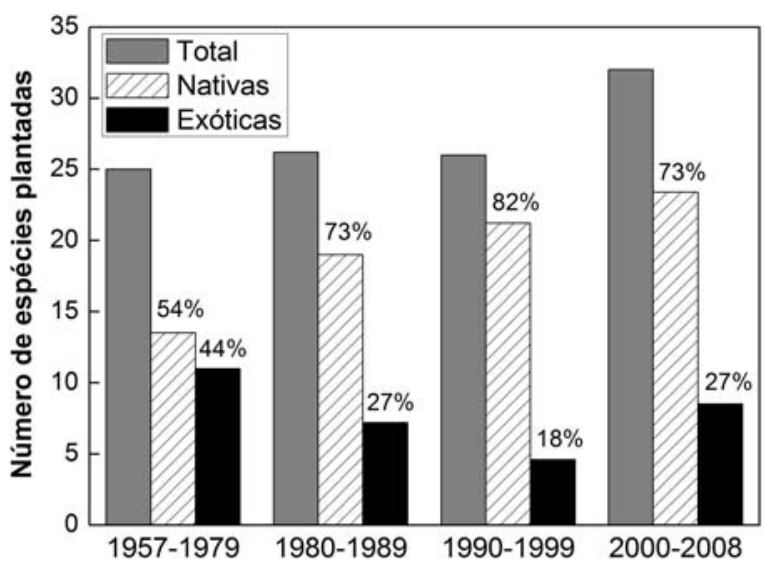

Figura 2 - Média do número total de espécies arbóreas plantadas (amostradas em $1.000 \mathrm{~m}^{2}$ ), e porcentagem de espécies nativas e exóticas utilizadas em plantios de restauração de matas ciliares no Estado de São Paulo, agrupados segundo a década de implantação dos projetos.

Figure 2-Mean of the total number of tree species (sampled in $1000 \mathrm{~m}^{2}$ ), and percentage of native and exotic species used in riparian forest restoration in the State of São Paulo, grouped according to decade of projects implementation.

Revista Árvore, Viçosa-MG, v.37, n.4, p.599-609, 2013 áreas seja responsável por uma pequena parte da variação no número de espécies nativas utilizadas ( $\mathrm{R}^{2}=0,13 ; \mathrm{p}<0,05$; Figura 1$)$, o número de espécies exóticas não é explicado pela idade dos plantios $\left(R^{2}=0,01 ; p=0,43, n . s\right)$.

Ao longo dos últimos 50 anos de restauração florestal no Estado de São Paulo, observamos que o número de espécies nativas utilizadas nos projetos aumentou, se analisados os plantios pela década em que foram implantados (Figura 2). O número total de espécies plantadas também aumentou nos anos mais recentes, passando de 25 espécies em média nas décadas de 1970, 1980 e 1990, para 33 espécies entre 2000 e 2008. Porém, o aumento no número médio de espécies nativas foi acompanhado pelo aumento no número de espécies exóticas, que vinha decrescendo até 1999 (1957-1979 = 11 espécies exóticas; 1980-1989 = 7; 1990-1999 = 5; 2000-2008 = 9; Figura 2) .

Por outro lado, a análise da freqüência de uso de espécies exóticas nos plantios evidencia que quanto maior o número de espécies plantadas, também tende a ser maior o número de espécies exóticas utilizadas para a restauração das matas ciliares (Figura 3). O número total de espécies plantadas explica 53\% da variação no número de espécies exóticas utilizadas.

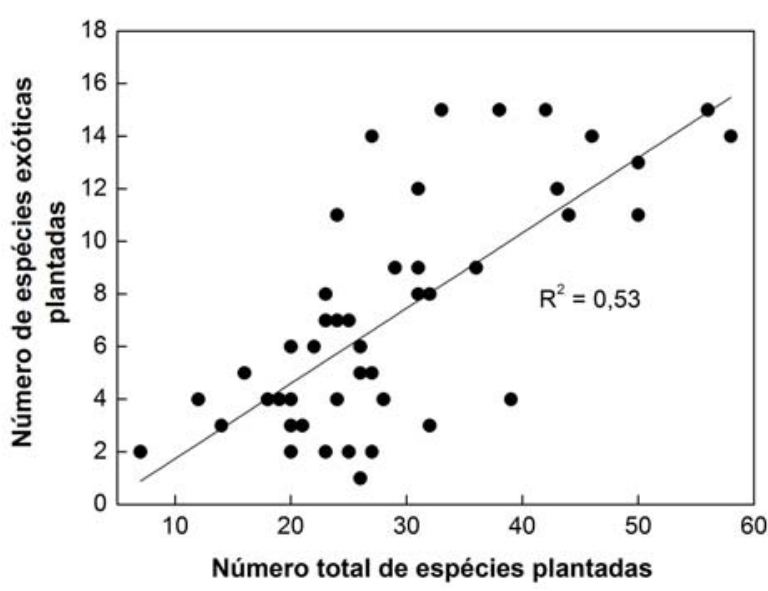

Figura 3 - Relação entre o número total de espécies arbóreas plantadas e o número de espécies exóticas utilizadas em plantios de restauração de matas ciliares. $\mathrm{R}^{2}$ : coeficiente de determinação.

Figure 3 -Relationship between the total number of species planted and number of exotic species used for riparian forests restoration. $R^{2}$ : determination coefficient. 


\section{DISCUSSÃO}

Ao longo dos últimos 50 anos de prática da restauração de matas ciliares no estado de São Paulo, espécies nativas vêm sendo cada vez mais priorizadas nos projetos, aumentando em número a cada década. No entanto, espécies exóticas foram e continuam a ser amplamente utilizadas na restauração. Com as mudanças ocorridas devido ao avanço do conhecimento e às restrições impostas pela legislação quanto ao uso exclusivo de espécies nativas, esperava-se que, especialmente nos últimos dez anos, espécies exóticas fossem ausentes ou ao menos ocasionais, o que não foi observado. Espécies exóticas estiveram presentes em $100 \%$ dos plantios estudados e em proporções elevadas, uma vez que uma em cada quatro espécies plantadas em matas ciliares na última década não era nativa da região de plantio.

Com relação à riqueza, o número de espécies plantadas registrado foi em geral baixo (28 espécies em cada área, em média). É possível que um número maior de espécies tenha sido plantado (inclusive de exóticas) e que muitas delas não tenham se adaptado ao ambiente restaurado, resultando resultando em menor riqueza amostrada do que no momento do plantio. Cabe ainda a ressalva de que número de espécies amostradas corresponde à área de apenas $1000 \mathrm{~m}^{2}$ em cada local. Mesmo com essas considerações, a riqueza amostrada está aquém do esperado para a restauração de florestas tropicais, que busca a máxima diversidade possível.

O aumento significativo do número de espécies utilizadas na restauração de matas ciliares na última década, em comparação com períodos anteriores, deve estar diretamente relacionado à pressão das exigências legais que passaram a vigorar a partir de 2001. Porém, esse aumento foi acompanhado por aumento também no número de espécies exóticas, que vinha diminuindo nas quatro décadas anteriores (até 1999). Se espécies exóticas são encontradas mesmo em plantios recentes, é porque mudas dessas espécies ainda são produzidas nos viveiros e destinadas aos projetos de restauração. De fato, verificamos que quanto maior o número de espécies usadas nos plantios, maior é a probabilidade de se introduzir também uma espécie exótica, aumentando o risco de invasões biológicas a partir das áreas restauradas.

A introdução de espécies exóticas em todo o mundo está diretamente relacionada, na maioria dos casos, com os interesses humanos, seja pelo potencial ornamental, de horticultura, para fins comerciais ou mesmo culturais (RICHARDSON; REJMÁNEK, 2011). No caso da restauração florestal, a utilização de espécies exóticas tem ligação histórica com o desenvolvimento dessa prática no Brasil, a começar pela restauração da Floresta da Tijuca (ABREU; RODRIGUES, 2010). A utilização de espécies exóticas tem ocasionado problemas para algumas regiões, com essas espécies escapando das áreas restauradas e invadindo áreas naturais remanescentes nas proximidades (ex. Leucena leucocephala, Tecoma stans, Schizolobium parahyba), de modo que alguns dos projetos de restauração florestal podem se configurar como uma das formas de disseminação dessas espécies em diferentes regiões de ocorrência da Mata Atlântica (BRANCALION et al., 2009).

Rodrigues et al. (2009) avaliaram que, durante os períodos iniciais da restauração, a produção e o uso de mudas de outras formações vegetacionais do Brasil era comum, sendo todas consideradas 'nativas'. Até hoje, essa percepção faz com que espécies de outras regiões sejam utilizadas sem restrições na maioria dos plantios de restauração. Mesmo que exista a consciência das peculiaridades fitogeográficas, em muitos casos, espécies de outras formações vegetacionais brasileiras são utilizadas nos plantios de restauração por falta de conhecimento sobre suas regiões de ocorrência natural. O erro também decorre, muitas vezes, da semelhança entre determinadas espécies exóticas e as nativas do mesmo grupo taxonômico. Uma espécie que ilustra bem essa situação é Acacia tenuifolia. Nativa da Floresta Estacional Decidual, essa espécie foi amostrada em três plantios efetuados pela Companhia Energética de São Paulo (CESP), tendo sido introduzida acidentalmente nos viveiros e nos plantios da empresa porque suas mudas são muito semelhantes às do angico (Anadenanthera macrocarpa) (Celso Machado, CESP, comunicação pessoal). A espécie só foi identificada corretamente quando da realização do presente estudo e, sem identificação correta, foi amplamente utilizada e agora se dissemina rapidamente entre as plantas em regeneração em vários locais. Também pela semelhança morfológica, a espécie da família Meliaceae Clausena excavata foi introduzida em Iracemápolis, confundida com Cabralea canjerana (Pedro H.S. Brancalion, comunicação pessoal), e hoje exerce forte dominância sobre espécies nativas no estrato regenerante.

A composição de espécies constitui aspecto fundamental de qualquer ecossistema, por ser determinante de sua estrutura e função (CLEWELL; 
ARONSON, 2007). Por isso, buscar a composição de espécies mais apropriada a cada ambiente que se pretenda restaurar é de grande importância. No entanto, a responsabilidade pela escolha das espécies geralmente cabe aos profissionais que elaboram projetos ou que atuam diretamente na restauração e que, muitas vezes, não possuem conhecimento adequado para a tarefa. A falta de fiscalização nos viveiros, aliada à dificuldade de identificação correta das espécies por parte dos viveiristas, coletores de sementes, agentes de órgãos licenciadores e demais profissionais que atuam na restauração estão entre os fatores responsáveis pela presença dessas espécies nos plantios. Dessa forma, a falta de treinamento e fiscalização, visando evitar o uso de espécies exóticas potencialmente invasoras, pode comprometer o sucesso de projetos de restauração. O risco de invasão deve ser sempre levado em consideração quando se trata de espécies não-nativas, as quais podem exibir comportamentos populacionais diferentes de acordo com os ambientes em que estão sendo introduzidas (COLAUTTI; MCISAAC, 2004; VAN KLEUNEN, et al. 2010).

A restauração ecológica de ecossistemas naturais busca, dentro de limites razoáveis, a maior semelhança possível entre a área restaurada e os ecossistemas naturais que foram destruídos. Partindo-se dessa premissa, a diminuição ou a supressão do uso de espécies exóticas nos projetos de restauração é algo desejável (HOBBS; RICHARDSON, 2011). Todavia, a destinação de recursos e esforços visando à erradicação generalizada de espécies já plantadas, simplesmente pelo fato de não serem nativas, não é recomendada (SIMBERLOFF et al., 2005; GARDENER et al., 2011). Iniciativas de manejo visando erradicar espécies exóticas devem ser sempre acompanhadas de estudos ecológicos específicos que demonstrem que a permanência dessas espécies pode colocar em risco o ecossistema em restauração ou até mesmo ecossistemas naturais vizinhos.

Poucos estudos sobre a restauração de ecossistemas têm enfocado espécies invasoras, exóticas ou indesejáveis, como analisado por Oliveira e Engel (2011). Assim, o papel ecológico que essas espécies têm desempenhado nas comunidades e nos ecossistemas ainda não foi devidamente elucidado (D’ANTONIO; MEYERSON, 2002). As espécies exóticas que têm sido utilizadas podem não ser ou não vir a se tornar invasoras nas áreas em que foram introduzidas (WILLIAMSON, 1996; DAWSON et al., 2009). Além disso, sabe-se que algumas espécies exóticas podem desempenhar papéis positivos nos ecossistemas, como, por exemplo, o de facilitadoras no estabelecimento de outras espécies ou na estruturação das comunidades (D’ANTONIO; MEYERSON, 2002; EWEL; PUTZ, 2004; MARTÍNEZ, 2010; SCHLAEPFER et al., 2011).

O avanço do conhecimento gerado pela comunidade científica e as políticas públicas ambientais no Estado de São Paulo que se destinaram a estabelecer normas rigorosas quanto às espécies a utilizar ainda não foram efetivas em restringir o uso de espécies exóticas em plantios de restauração. Para promover plantios mais adequados aos objetivos da restauração ecológica e que primem por espécies nativas, é necessário intensificar a fiscalização na produção das mudas fornecidas pelos viveiros e oferecer treinamento adequado aos profissionais ligados à restauração ecológica em todas as suas etapas. Além disso, é preciso intensificar as pesquisas. Estudos de longo prazo sobre o desenvolvimento das florestas em restauração e a maneira como as espécies exóticas se comportam nessas comunidades são necessários, como ferramentas de suporte às decisões de manejo relativas às espécies exóticas que já existam ou que venham a ser utilizadas na restauração ecológica ou na reabilitação de ecossistemas degradados.

\section{AGRADECIMENTOS}

À Secretaria do Meio Ambiente do Estado de São Paulo, pelo financiamento do projeto de pesquisa Trajetórias Sucessionais de Plantios de Restauração de Matas Ciliares, no âmbito do Projeto Mata Ciliar, à Fundação de Amparo à Pesquisa no Estado de São Paulo-FAPESP, pela bolsa concedida a G.B.A. (Processo 2009/11752-8), ao Conselho Nacional de Desenvolvimento Científico e Tecnológico - CNPq, pela bolsa de doutorado a M.S.S. (Processo 143423/2009-6) e de produtividade em pesquisa a G.D. (Processo 303402/2012-1).

\section{REFERÊNCIAS}

ABREU, R. C. R.; RODRIGUES, P. J. F. P. Exotic tree Artocarpus heterophyllus (Moraceae) invades the Brazilian Atlantic Rainforest. Rodriguésia, v. 61, n. 4, p. 677-688, 2010. 
ARONSON, J. et al. What Role Should Government Regulation Play in Ecological Restoration? Ongoing Debate in São Paulo State, Brazil. Restoration Ecology, v. 19, n. 6, p. 690-695, 2011.

ARONSON, J. What can end Should Be Legalized in Ecological Restoration? Revista Árvore, v.34, n.3, p.451-454, 2010.

BARBOSA, L. M. et al. Recuperação florestal com espécies nativas no estado de São Paulo: pesquisas apontam mudanças necessárias. Florestar Estatístico, v.6, n.1, p.28-34, 2003.

BRANCALION, P.H.S. et al. Instrumentos legais podem contribuir para a restauração de florestas tropicais biodiversas. Revista Árvore, v.34, n.3, p.455-470, 2010.

BROCKERHOFF, E. G. et al. Plantation forests and biodiversity: oxymoron or opportunity?

Biodiversity and Conservation, v.17, p.925-951, 2008.

CARVALHO, P. E. R. Espécies arbóreas brasileiras. Brasília, DF: Embrapa Informação Tecnológica, 2003. v.3. 604p.

CHAZDON, M. Beyond deforestation: restoring forests and ecosystem services on degraded lands. Science, v.320, p.1458-1460, 2008.

CLEWELL, A. F.; ARONSON, J. Ecological restoration: principles, values, and structure of an emerging profession. Washington, DC: Island Press, 2007. 232p.

COLAUTTI, R. I.; MACISAAC, H. J. A neutral terminology to define 'invasive' species.

Diversity and Distributions, v.10, n.1, p.135-141, 2004.

CORRÊA, M. P. Dicionário das plantas úteis do Brasil e das exóticas cultivadas. Rio de Janeiro: IBDF, 1984. v.6.

D’ANTONIO, C. M.; MEYERSON, L. A. Exotic plant species as problems and solutions in ecological restoration: a synthesis. Restoration Ecology, v.10, n.4, p.703-713, 2002.
DAVIS, M. A. Invasion biology. New York: Oxford, 2009. 259 p.

DURIGAN, G. et al. A Flora arbustivo-arbórea do Médio Paranapanema: base para a restauração dos ecossistemas naturais. In: VILAS BÔAS, O. V.; DURIGAN, G. (Org.). Pesquisas em conservação e recuperação ambiental no Oeste Paulista: resultados da cooperação Brasil/Japão. São Paulo: Páginas \& Letras, 2004. p.199-239.

DURIGAN, G. et al. Normas jurídicas para a restauração ecológica: uma barreira a mais a dificultar o êxito das iniciativas? Revista Árvore, v.34, n.3, p.471-485, 2010.

DURIGAN, G.; DIAS, H. C. S. Abundância e diversidade da regeneração natural sob mata ciliar implantada. In: CONGRESSO FLORESTAL BRASILEIRO, 68., 1990, Campos do Jordão. Anais... Campos do Jordão: Sociedade Brasileira de Silvicultura, 1990. p.308-312.

EWEL, J. J.; PUTZ, F. E. A place for alien species in ecosystem restoration. Frontiers in Ecology and the Environment, v.2., p.354-360, 2004.

GARDENER, M. R. et al. Plant invasions research in Latin America: fast track to a more focused agenda. Plant Ecology \& Diversity, v.1, n.1, p.1-8, 2011.

HOBBS, R. J.; RICHARDSON, D. M. Invasion ecology and restoration ecology: Parallel evolution in two fields of endeavour. In: RICHARDSON, D. M. (Ed.). Fifty years of invasion ecology: The Legacy of Charles Elton. Oxford: Blackwell Publishing, 2011. p.61-69.

van KLEUNEN, M. et al. Are invaders different? A conceptual framework of comparative approaches for assessing determinants of invasiveness.

Ecology Letters, v.13, p.947-958, 2010.

LORENZI, H. Árvores brasileiras: manual de identificação e cultivo de plantas arbóreas do Brasil. Nova Odessa, Plantarum, 1992. 352p.

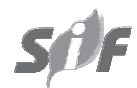

Revista Árvore, Viçosa-MG, v.37, n.4, p.599-609, 2013 
MACK, R.N. et al. Biotic invasions: causes, epidemiology, global consequences and control. Ecological Applications, v.10, p.689-710, 2000.

MELO, A. C. G.; DURIGAN, G. Evolução estrutural de reflorestamentos de restauração de matas ciliares no Médio Vale do Paranapanema.

Scientia Forestalis, n.73, p.101-111, 2007.

MITTERMEIER, R. A., et al. Hotspots revisited: earth's biologically richest and most endangered terrestrial ecoregions. Washington, DC: Cemex, 2004. 392p.

NOGUEIRA, J. C. B. Reflorestamento heterogêneo com essências indígenas. São Paulo: Instituto Florestal, 1977. p.1-17. (Boletim Técnico, 24).

OLIVEIRA, R. E.; ENGEL, V. L. A restauração ecológica em destaque: um retrato dos últimos vinte e oito anos de publicações na área. Oecologia Australis, v.15, n.2, p.303-315, 2011.

PALMER, M. A.; AMBROSE, R. F.; POFF, N. L. Ecological theory and commmunity restoration ecology. Restoration Ecology, v.5, n.4, p.291-300, 1997.

PARROTTA, J. A.; KNOWLES, O. H. Restoration of tropical moist forests on bauxite-mined lands in the Brazilian Amazon. Restoration Ecology, v.7, p.103-116, 1999.

PRACH, K. et al. Manipulation of succession. In: WALKER, L. R.; WALKER, J.; HOBBS, R. J. (Ed.). Linking restoration and ecological succession. New York: Springer, 2007. p.121-149.

PULITANO, F. M.; DURIGAN, G.; DIAS, L. E. A mata ciliar da Fazenda Cananéia: estrutura e composição florística em dois setores com idades diferentes. In: BÔAS, O.V.; DURIGAN, G. (Org.).

Pesquisas em conservação e recuperação ambiental no Oeste Paulista: resultados da cooperação Brasil/Japão. São Paulo: Páginas e Letras, 2004. v.1. p.419-445.

RAMOS, V. S. et al. Árvores da Floresta Estacional Semidecidual. São Paulo: EDUSP/FAPESP, 2008. v.1. 320 p.
REJMANEK, M.; RICHARDSON, D.M.; PYSEK, P. Plant invasions and invasibility of plant communities. In: van der MAAREL, E. (Ed.). Vegetation ecology. Oxford: Blackwell Publishing, 2005. p.332-355.

RICHARDSON, D. M.; REJMÁNEK, M. Trees and shrubs as invasive alien species: a global review. Diversity and Distributions, v.17, p.788-809, 2011.

RODRIGUES, E. R.; MONTEIRO, R.; CULLEN JUNIOR, L. Dinâmica inicial da composição florística de uma área restaurada na região do Pontal do Paranapanema. Revista Árvore, v.34, n.5, p.853-861, 2010.

RODRIGUES, R. R. et al. On the restoration of high diversity forests: 30 years of experience in the Brazilian Atlantic Forest. Biological Conservation, v.142, p.1242-1255, 2009.

RODRIGUES, R. R.; NAVE, R. R. Heterogeneidade florística das matas ciliares. In: RODRIGUES, R. R.; LEITÃO-FILHO, H. F. (Org.). Matas ciliares: conservação e recuperação. São Paulo: EDUSP/FAPESP, 2000. p.159-167.

SÃO PAULO. Resolução SMA 08, de 31 de janeiro de 2008. Fixa a orientação para o reflorestamento heterogêneo de áreas degradadas e dá providências correlatas. Diário Oficial do Estado de São Paulo, São Paulo, p. 31. 1 fev. 2008. Seção 1.

SCHLAEPFER, M. A.; SAX, D. F.; OLDEN, J. D. The potential conservation value of non-native species. Conservation Biology, v.25, p.428-437, 2011.

SOCIETY FOR ECOLOGICAL RESTORATION INTERNATIONAL SCIENCE - SER. Policy Working Group. 2004. The SER primer in ecological restoration (Version 2). Tucson: 2009. Disponível em: URL: http://www.ser.org/ content/ecological_restoration_primer.asp [accessed on $10 \mathrm{Feb}$. 2009]

SIMBERLOFF, D.; PARKER, I. M.; WINDLE, P.N. Introduced species policy, management, and future research needs. Frontiers in Ecology and Environment, v.3, n.1, p.12-20, 2005. 
SOUZA, F. M.; BATISTA, J. L. F. Restoration of seasonal semideciduous forests in Brazil: Influence of age and restoration design on forest structure. Forest Ecology and

Management, v.191, p.185-200, 2004.

VELOSO, H. P.; RANGEL FILHO, A. L.; LIMA, J. C. A. Classificação da vegetação brasileira adaptada a um sistema universal. Rio de Janeiro: IBGE, 1991. 124 p.

WANDERLEY, M. G. L. et al. (Coord.) Flora Fanerogâmica do Estado de São Paulo. São Paulo: Instituto de Botânica, 2009. v.6.

WANDERLEY, M. G. L. et al. (Coord.). Flora Fanerogâmica do Estado de São Paulo. São Paulo: FAPESP-RIMA, 2005. v.4.
WANDERLEY, M. G. L. et al. (Coord.). Flora Fanerogâmica do Estado de São Paulo. São Paulo: FAPESP-RIMA, 2003. v.3.

WANDERLEY, M. G. L.; SHEPERD, G. J.; GIULIETTI, A. M. (Coord.). Flora Fanerogâmica do Estado de São Paulo. São Paulo: FAPESP-HUCITEC, 2001. v.1.

WANDERLEY, M. G. L.; SHEPERD, G. J.; GIULIETTI, A. M. (Coord.). Flora

Fanerogâmica do Estado de São Paulo. São Paulo: FAPESP-HUCITEC, 2002. v.2.

WANDERLEY, M. G. L. et al. (Coord.). Flora Fanerogâmica do Estado de São Paulo. São Paulo: Imprensa Oficial, 2007. v.5. 
